\title{
Orbital and Charge-Resolved Polaron States in CdSe Dots and Rods Probed by Scanning Tunneling Spectroscopy
}

\author{
Zhixiang Sun (孙志祥), ${ }^{1}$ Ingmar Swart, ${ }^{1,2}$ Christophe Delerue,${ }^{3}$ Daniël Vanmaekelbergh, ${ }^{1}$ and Peter Liljeroth ${ }^{1, *}$ \\ ${ }^{1}$ Condensed Matter and Interfaces, Debye Institute for Nanomaterials Science, University of Utrecht, \\ PO Box 80000, 3508 TA Utrecht, The Netherlands \\ ${ }^{2}$ Inorganic Chemistry and Catalysis, Debye Institute for Nanomaterials Science, University of Utrecht, \\ Sorbonnelaan 16, 3584 CA Utrecht, The Netherlands \\ ${ }^{3}$ Institut d'Electronique, de Microélectronique et de Nanotechnologie (IEMN), Département ISEN, \\ 41 bd Vauban, 59046 Lille Cedex, France
}

(Received 4 March 2009; published 12 May 2009)

\begin{abstract}
Conduction electrons interact with lattice vibrations, leading to coupled electron-phonon states. This effect is of fundamental importance in understanding electron transport and energy relaxation in nanoscale systems. We report quantitative results on the electron-phonon coupling strength in CdSe quantum dots (QDs) and rods, obtained by low-temperature scanning tunneling microscopy. We resolve the polaron eigenstates arising from coupling of the electrons to longitudinal optical phonons. The electron-phonon coupling strength is dependent on the electron orbital symmetry, the number of added electrons, and the size and dielectric environment of the QD.
\end{abstract}

DOI: 10.1103/PhysRevLett.102.196401

PACS numbers: 71.38.- $\mathrm{k}, 73.22 .-\mathrm{f}, 73.63 .-\mathrm{b}$

Colloidal semiconductor quantum dots (QDs) have wellseparated energy levels due to quantum confinement, giving these systems unique optical and electrical properties [1-6]. As a result, a large variety of applications based on these materials has been proposed, ranging from biomarkers to photovoltaics $[1,2,7,8]$. For all proposed applications of semiconductor QDs, electron transport and energy relaxation are important processes that need to be understood. The charge of a conduction electron in a semiconductor leads to a local lattice distortion, i.e., a polaron. This distortion is enhanced in nanostructures due to confinement of the electron wave function [5,912]. As a result, the density of states (DOS) in a nanostructured semiconductor is qualitatively different from the simple particle-in-a-box picture. Electron-phonon ( $e$-ph) coupling is therefore central to our understanding of transport and energy relaxation in nanoscale systems [1-5,1318]. For example, once competing relaxation processes have been minimized by nanocrystal engineering, coupling of the electron to lattice vibrations is the only remaining relaxation pathway, as it is an intrinsic process [2]. In addition, $e$-ph coupling underpins the temperature dependence of electron transport in QD superlattices [19,20].

Despite an abundance of theoretical results [5,9-11], clear-cut experimental data regarding $e$-ph coupling in macroscopic and low-dimensional semiconductors is lacking $[12,21,22]$. Obvious questions, not yet answered, deal with the relationship between the symmetry and extension of electronic orbitals, the crystal shape and its dielectric environment, and the $e$-ph coupling strength. We address these questions by measuring the single-electron DOS of semiconductor QDs with scanning tunneling microscopy (STM) and spectroscopy (STS) under conditions where ground and excited polaron states can be observed.
Previous experiments on individual defects, dangling bonds, and molecules have demonstrated that STM can be used to quantitatively probe individual polaron levels down to the atomic scale [23-25].

Here, we present STS results on spherical and rodshaped CdSe QDs. We measure the single-particle DOS under shell-tunneling $[5,26]$ and derive the $e$-ph coupling strength for different orbital symmetries. In the shell-filling regime $[5,26]$, we probe the effects of an additional added electron. Our results show the formation of polaron eigenstates arising from Fröhlich coupling of an electron to longitudinal optical (LO) phonons. The coupling strength is found to depend considerably on the orbital symmetry, the number of added electrons, and on the size and dielectric environment of the nanocrystal.

We have carried out low-temperature STM experiments on arrays of colloidal CdSe QDs (3 and $6 \mathrm{~nm}$ in diameter) and mixtures of dot- and rod-shaped QDs (diameter of $3.5 \mathrm{~nm}$ and an aspect ratio between 1 and 4). The QDs were synthesized and deposited on highly oriented pyrolytic graphite (HOPG) or flame-annealed gold substrates as described previously [12,27,28]. STM experiments (LT STM, Omicron Nanotechnology) were carried out at $T=4.8 \mathrm{~K}$ with cut PtIr tips (typical bias of $2.5 \mathrm{~V}$ and setpoint current of $10 \mathrm{pA}$ ). The tunneling spectra were acquired at decreased bias and increased set-point (typically $1.5 \mathrm{~V} / 100-200 \mathrm{pA}$ ). The tunneling conductance was recorded simultaneously using a lock-in amplifier (amplitude $3 \mathrm{mV}$ rms. at a frequency of $300 \mathrm{~Hz}$ ). We performed STS at several samples (HOPG and Au substrates, spherical and rod-shaped QDs); in total ca. 100 QDs were investigated. A typical large scale STM image of the rod-shaped QDs on HOPG is shown in Fig. 1(a). We can resolve the size and shape of the QDs and select any of them for STS. 
As shown previously, CdSe QDs do not show significant quantum mechanical coupling and hence, even in an array, the measured spectra reflect the DOS of individual QDs [28]. A tunneling spectrum ( $d I / d V$ vs $V$ ) measured on a spherical QD is shown in Fig. 1(b); we immediately notice the progression of peaks corresponding to each of the quantum confined energy levels. These peaks are equally spaced and strongly resemble the expected response when the electron level is coupled to phonon modes [5,9]. This coupling between the confined electron and a lattice deformation results in the formation of a polaronic DOS, where each one-electron configuration is characterized by a ground and several vibrationally excited states, separated by the phonon energy.

The double-barrier tunnel junction shown in Fig. 1(c) is characterized by the tunneling rates into, $\Gamma_{\text {in }}$, and out of, $\Gamma_{\text {out }}$, the QD $[16,26]$. The ratio $\Gamma_{\text {in }} / \Gamma_{\text {out }}$ will determine the number of additional electrons in the QD. In the limiting case $\Gamma_{\text {in }} \ll \Gamma_{\text {out }}$, i.e., under shell-tunneling conditions, electrons tunnel through the QD one by one and $e-e$ interactions do not occur. In this case, the peaks in the

(a)

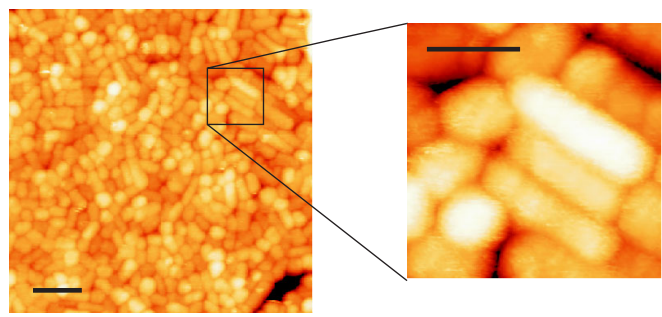

(b)
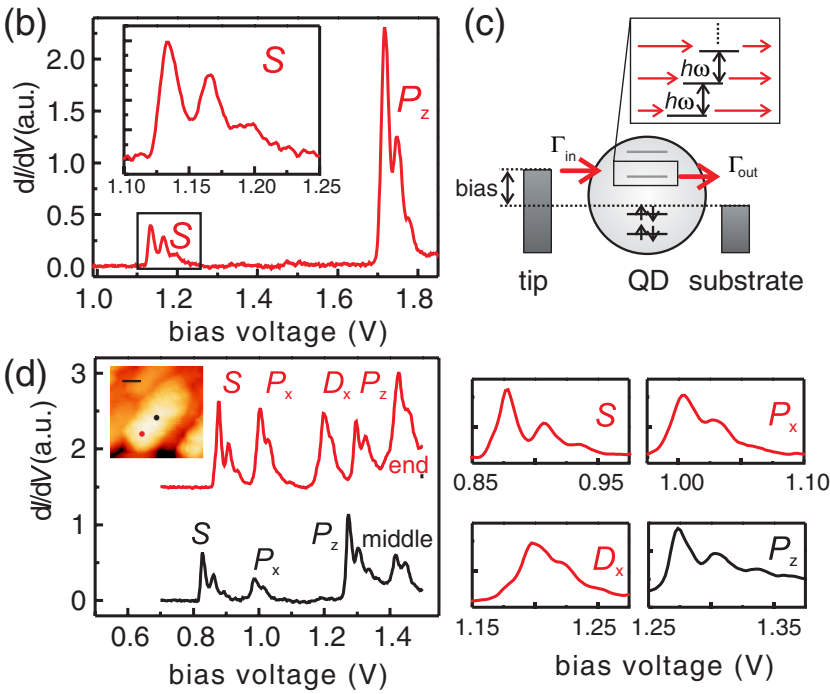

FIG. 1 (color online). (a) STM topography of arrays of colloidal CdSe QDs on a HOPG substrate. Scale bars 20 and $10 \mathrm{~nm}$ (inset). (b) Spectroscopy on a spherical QD with a diameter of $3 \mathrm{~nm}$. Inset: zoom-in to the first resonance that corresponds to tunneling through the $S$ level. (c) Schematic illustration of the transport processes in a double-barrier tunnel junction with polaronic DOS. (d) Spectroscopy on a CdSe nanorod with an aspect ratio of ca. 2.5 showing identification of the levels based on spatially resolved measurements. The positions where the spectra were recorded are indicated in the inset (scale bar $3 \mathrm{~nm}$ ). tunneling spectrum reflect the polaronic DOS, and the $e$-ph coupling strength can be directly quantified based on the amplitudes of the elastic peak and the replica.

The dependence of the $e$-ph coupling strength on the orbital symmetry can be conveniently studied in rodshaped QDs. The crystal shape asymmetry along the $x$ axis will break the degeneracy of the $P$ (and higher lying) levels and cause the $P_{x}$ state to decrease in energy with respect to $P_{y}$ and $P_{z}$ states. Depending on the aspect ratio of the nanorod, the spectrum can be rather complex with for example $D_{x}$ and $P_{z}$ states having similar energy. These states can be distinguished by probing their symmetry with spatially resolved spectroscopy experiments $[29,30]$ as illustrated in Fig. 1(d): just below the rod midpoint, we are close to a node of the $P_{x}$ and $D_{x}$ states, while the $P_{z}$ state has significant weight. Conversely, at the end of the rod, resonances corresponding to $P_{x}$ and $D_{x}$ states have higher amplitude than the one corresponding to the $P_{z}$ state. As can be seen in Fig. 1(d), we can resolve the phonon replica of all the levels and hence, directly quantify the $e$-ph coupling strength for orbitals with different symmetry (vide infra).

In the case of a single phonon mode, theory predicts a series of equally spaced peaks (spacing $\hbar \omega$ ) with the intensity of the $n$th peak being proportional to $g^{n} / n$ !, where $g$ is the Huang-Rhys factor, i.e., the dimensionless $e$-ph coupling strength [5,9]. For comparison with experiment, the theoretical response is broadened with Gaussians. We show two examples in Fig. 2(a); in both cases, there is quantitative agreement between theory and experiment. The fitting yields spacings of the replica of 34 and $33 \mathrm{mV}$, coupling strengths $g$ of 0.35 , and 0.62 for the dot and rod, respectively.

There is a small but systematic difference between the theoretical predictions and the experimental results. We always observe the first resonance to be slightly asymmetric and broader on the high bias side, see Fig. 2(a). This is likely to be caused by coupling to other (acoustic) phonon modes with smaller energies. Our energy resolution is not sufficient to resolve these phonons; hence, they only lead to a broadening of the resonances. In addition, the replicas are broader than the elastic resonance; this might indicate some dispersion in the phonon energies or lifetime broadening of the vibrationally excited states due to the presence of rapid relaxation processes (in the sub-ps time scale).

We show a histogram of the measured peak spacings over several samples in Fig. 2(b). The distribution is strongly peaked around $31 \mathrm{mV}$. In order to convert this number to the true energy scale, the potential distribution in the tip-QD-substrate junction has to be taken into account. Based on the solution of the Poisson equation for a realistic tip-QD-substrate geometry [5,26,28], ca. $85 \%$ of the bias voltage drops over the tip-QD junction, and hence, the bias voltage has to be multiplied by 0.85 to convert it to the energy scale. This gives an energy spacing of $26 \mathrm{meV}$, in excellent agreement with the known LO phonon energy 

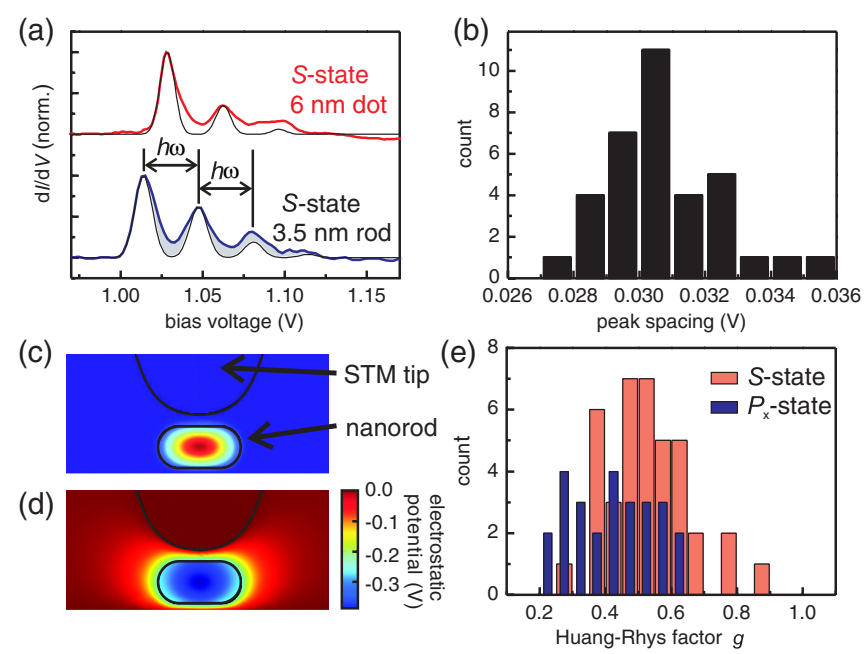

FIG. 2 (color online). Analysis of the $e$-ph coupling strength. (a) Experimental data for $6 \mathrm{~nm}$ spherical dot and $3.5 \mathrm{~nm}$ diameter nanorod (aspect ratio ca. 2) along with the fits to a theory with a coupling strength of $g=0.35$ (dot) and $g=0.62$ (rod). (b) Distribution of the spacing of the phonon replica. (c) Solution of the Schrödinger equation for a nanorod (diameter $3.5 \mathrm{~nm}$, aspect ratio 2) in the STM-substrate junction. (d) The resulting electrostatic potential distribution based on the solution of the Poisson equation. (e) $e$-ph coupling strength for the $S$ and $P_{x}$ states measured on several nanorods.

of bulk CdSe [31]. There is further theoretical evidence that the electrons indeed couple to the LO phonons. For a spherical QD, the coupling strength can be estimated analytically in the effective mass approximation $[5,11,12]$. Numerical simulations can be used to take into account the effects of finite confining potential, orbital symmetry, dot shape and dielectric environment on $g$. The coupling strength for this so-called Fröhlich coupling is given by $g=d_{\mathrm{FC}} / \hbar \omega$, where $d_{\mathrm{FC}}$ is the relaxation energy of the crystal lattice due to the addition of the electron, i.e., formation of a polaron. This energy can be estimated as $d_{\mathrm{FC}}=1 / 2\left\{\left[\int \rho(\mathbf{r}) \phi(\mathbf{r}) d \mathbf{r}\right]_{\infty}-\left[\int \rho(\mathbf{r}) \phi(\mathbf{r}) d \mathbf{r}\right]_{0}\right\}$, where $\rho$ is the charge density due to the additional electron and $\phi$ is the corresponding electrostatic potential, evaluated either at the high frequency $(\infty)$ or static $(0)$ limit [5]. $d_{\mathrm{FC}}$ can be numerically estimated by first solving the Schrödinger equation for a particle in a finite box to obtain the charge density as illustrated in Fig. 2(c). This charge density gives rise to an electrostatic potential distribution [Fig. 2(d)], which is obtained as a solution of the Poisson equation, either using the high frequency or static dielectric constants. Despite the known problems of the effective mass approach, this procedure is expected to give a good approximation of the $e$-ph coupling strength as it involves a volume integral that is insensitive to the details of the wave function and the response of the ions to the local electric field is not affected by quantum confinement effects. The calculation is done in a realistic STM junction geometry to include the screening from the metallic tip and the substrate [32]. Ignoring the screening from the neighboring
QDs, this gives an estimate for the coupling strengths of 0.42 (rod) and 0.36 (dot), in fair agreement with the experimental results shown in Fig. 2(a).

The effect of the orbital symmetry on the coupling strength is summarized in Fig. 2(e) for the $S$ and $P_{x}$ states for several nanorods with different aspect ratios. There is a significant difference in the coupling strength between the $S$ and $P_{x}$ states (average values $g=0.53$ and 0.42 , respectively). We observe (both experimentally and theoretically) only a weak dependence of $g$ on the nanorod aspect ratio (not shown). The widths of the distributions shown in Fig. 2(e) reflect significant variations in the coupling strengths. In principle, the $e$-ph coupling is an intrinsic effect. However, the extension of the electrostatic potential distribution outside the nanorod makes the coupling strength sensitive to the dielectric environment of the QD. As limiting cases, we consider theoretically an isolated QD and a QD embedded in a slab of dielectric (same $\epsilon_{0}$ and $\epsilon_{\infty}$ as CdSe) in the STM junction geometry. The calculated values for a $3.5 \mathrm{~nm}$ diameter nanorod with an aspect ratio of 2 are $g=0.42$ (isolated) and 0.71 (slab) for the $S$ state and $g=0.29$ (isolated) and 0.60 (slab) for the $P_{x}$ state. Thus, the variation in the dielectric environment of the QDs can fully explain both the average and the spread of the experimentally observed values.

Typically, due to the asymmetric junction geometry, $\Gamma_{\text {in }}<\Gamma_{\text {out }}$ as the set-point current is limited to $<1 \mathrm{nA}$ (higher currents cause instabilities in the double-barrier tunnel junction). However, in some cases, the QD is weakly coupled with the substrate, making it possible to reach $\Gamma_{\text {in }}>\Gamma_{\text {out }}$. In this case, the degeneracy of the levels is lifted due to interactions between the carriers accumulating in the QD. An example of this is shown in Fig. 3(a): as the set-point current is increased, we observe a new resonance at a bias of ca. 1.2 V. This corresponds to the addition of a second electron into the $S$ orbital of the QD and the spacing between the $S(1,0)$ and $S(2,0)$ resonances [see Figs. 3(b) and 3(c) for the labeling of the resonances] is equal to the $e-e$ repulsion $\left(J_{\mathrm{ee}}\right), 55 \mathrm{meV}$ in the present case. The spectra acquired at the highest and lowest set points, normalized by the amplitude of the first resonance, are shown in Fig. 3(c). The relative amplitudes of the $S(1,0)$ and $S(2,0)$ resonances give information on the ratio of $\Gamma_{\text {in }} / \Gamma_{\text {out }}$; in the limit of $\Gamma_{\text {in }} \gg \Gamma_{\text {out }}$, the amplitudes are equal as in the measured spectra at the highest set-point currents.

In the shell-filling regime, the current is determined by $\Gamma_{\text {out }}$. The evolution of the peak amplitudes as a function of the set-point current can be estimated by a master equation approach $[5,33]$. We consider transport through the $S(1,0)$, $S(1,1)$, and $S(2,0)$ states and calculate the current as a function of $\Gamma_{\text {in }}$ and $\Gamma_{\text {out }}$. The predictions for the $S(1,1)$ and $S(2,0)$ peak amplitudes are given in Fig. 3(d) along with the experimental results. The observed phonon replica has a strongly enhanced amplitude compared with theory. While this difference might be partially due to incomplete shell-filling, it cannot explain the increase of the $S(2,1)$ 


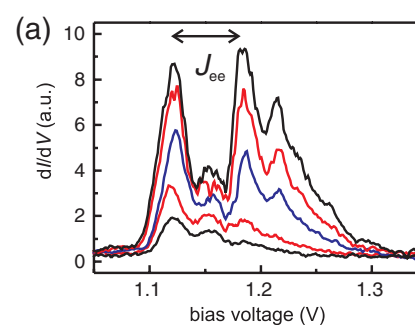

(b)
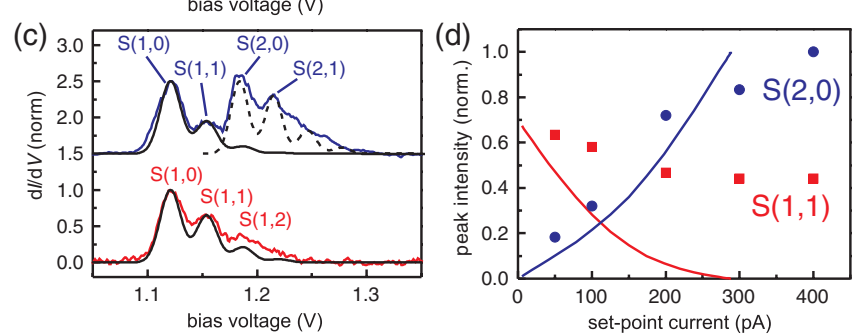

FIG. 3 (color online). Electron-phonon coupling under shellfilling conditions. (a) Evolution of the spectra from shelltunneling to shell-filling when the set-point is increased (50, $100,200,300$, and $400 \mathrm{pA}$ ). (b) Level occupations corresponding to the first 3 resonances. (c) Fitting of the spectra shows that the amplitude of the $e$-ph replica is enhanced for the second added electron. The labeling for the different resonances is indicated. (d) Evolution of the peak intensities with the set-point current: for the first phonon replica $[S(1,1)$, squares], for the addition of second electron $[S(2,0)$, circles] along with the corresponding theoretical predictions (solid lines).

amplitude with increasing set-point current. Understanding this significant enhancement of $e$-ph coupling in the shellfilling regime will require a more elaborate theoretical analysis, including the possible effects of nonequilibrium phonon population and the interplay between $e-e$ and $e$-ph interactions $[13,22]$.

In summary, our experiments provide quantitative orbital and charge-resolved information on the $e$-ph interaction in CdSe QDs. It is shown that Fröhlich coupling to the LO phonon mode is the dominant mechanism. The dependence of the coupling strength on the QD shape and size, the symmetry of the electronic orbital, and the dielectric environment of the QD can be understood within the effective mass and continuum dielectric picture. Our results provide fundamental information required in understanding electron transport in QD devices and superlattices $[3,19,20]$ and the intraband relaxation of hot carriers in QDs $[1,2,17,18]$.

We thank Rolf Koole for providing the nanorod samples used in this study. This research was supported by the EU (ITN "HERODOT") and NWO (Chemical Sciences, Vidigrant No. 700.56.423).
*P.Liljeroth@uu.nl

[1] V. I. Klimov, Annu. Rev. Phys. Chem. 58, 635 (2007).

[2] A. Pandey and P. Guyot-Sionnest, Science 322, 929 (2008).

[3] D. V. Talapin and C. B. Murray, Science 310, 86 (2005).

[4] U. Banin et al., Nature (London) 400, 542 (1999).

[5] C. Delerue and M. Lannoo, Nanostructures; Theory and Modelling (Springer, Berlin, 2004).

[6] J. J. Urban et al., Nature Mater. 6, 115 (2007).

[7] G. Konstantatos et al., Nature (London) 442, 180 (2006).

[8] I. L. Medintz et al., Nature Mater. 4, 435 (2005).

[9] N.S. Wingreen, K. W. Jacobsen, and J. W. Wilkins, Phys. Rev. B 40, 11834 (1989).

[10] S. Hameau et al., Phys. Rev. Lett. 83, 4152 (1999).

[11] D. V. Melnikov and W. B. Fowler, Phys. Rev. B 63, 165302 (2001).

[12] L. Jdira et al., Phys. Rev. B 77, 205308 (2008).

[13] B. J. LeRoy et al., Nature (London) 432, 371 (2004).

[14] D. L. Klein et al., Nature (London) 389, 699 (1997).

[15] D. Yu, C. J. Wang, and P. Guyot-Sionnest, Science 300, 1277 (2003).

[16] E. P. A. M. Bakkers et al., Nano Lett. 1, 551 (2001).

[17] R. D. Schaller et al., Nano Lett. 6, 424 (2006).

[18] E. Hendry et al., Phys. Rev. Lett. 96, 057408 (2006).

[19] A. J. Houtepen, D. Kockmann, and D. Vanmaekelbergh, Nano Lett. 8, 3516 (2008).

[20] D. Yu, C. Wang, B. L. Wehrenberg, and P. Guyot-Sionnest, Phys. Rev. Lett. 92, 216802 (2004).

[21] G. S. Boebinger et al., Phys. Rev. Lett. 65, 235 (1990).

[22] B. A. Carpenter et al., Phys. Rev. B 74, 161302(R) (2006).

[23] J. Repp et al., Phys. Rev. Lett. 95, 225503 (2005).

[24] M. Berthe et al., Science 319, 436 (2008).

[25] S. W. Wu et al., Phys. Rev. Lett. 93, 236802 (2004).

[26] P. Liljeroth et al., Phys. Chem. Chem. Phys. 8, 3845 (2006).

[27] C. de Mello Donegá et al., J. Phys. Chem. B 107, 489 (2003).

[28] L. Jdira et al., Nano Lett. 8, 4014 (2008).

[29] T. Maltezopoulos et al., Phys. Rev. Lett. 91, 196804 (2003).

[30] O. Millo et al., Phys. Rev. Lett. 86, 5751 (2001).

[31] II-VI and I-VII Compounds; Semimagnetic Compounds, Landolt-Börnstein-Group III Condensed Matter, edited by U. Rössler (Springer-Verlag, Berlin, 1999), Vol. 41.

[32] The calculations were performed using the finite element method (COMSOL Multiphysics 3.4) with typical mesh of $>150000$ elements. Parameters used were: barrier outside the QD $V_{0}=4 \mathrm{eV}$, effective electron mass $m_{\mathrm{eff}}=0.11$, $\epsilon_{\infty}=6.1, \epsilon_{0}=9.3$.

[33] We assume (bias dependent) tunneling rates of $\Gamma_{\text {in }}$ and $\Gamma_{\text {out }}$ for $S(1,0)$ and $S(2,0)$ transitions and $g \Gamma_{\text {in }}$ and $g \Gamma_{\text {out }}$ out for $S(1,1)$ transition. The simulations assume rapid internal relaxation from $S(1,1)$ to $S(1,0)$; however, assuming no internal relaxation has only a minor effect on the predicted peak amplitudes. 